\title{
Full genome analysis of circulating DENV-2 in Senegal reveals a regional diversification into separate clades
}

\begin{abstract}
Idrissa Dieng1 ${ }^{1}$, Amadou Diallo², Mignane Ndiaye ${ }^{1}$, Moussa Moïse Diagne ${ }^{1}$, Safietou Sankhe ${ }^{1}$ , Marie Henriette Dior Ndione ${ }^{1}$, Amadou Alpha Sall ${ }^{1}$, Cheikh Loucoubar ${ }^{2}$, Ousmane Faye ${ }^{1}$, Oumar Faye ${ }^{1}$
\end{abstract}

\begin{abstract}
${ }^{1}$ Arboviruses and Haemorrhagic Fever Viruses Unit, Virology Department, Institut Pasteur de Dakar, Dakar, Senegal

2 Epidemiology, Clinical Research and Data Science Department, Institut Pasteur de Dakar, Dakar, Senegal
\end{abstract}

\begin{abstract}
:
To assess the genetic diversity of circulating dengue virus 2 in Senegal in 2018 we performed molecular characterization by complete genome sequencing and performing phylogenetic analysis. Sequenced strains belong to Cosmopolitan genotype of DENV-2 we observed intragenotype variability leading to a divergence in two clades with differential geographic distribution. We report two variants namely; the "Northern variant" harbouring three nonsynonymous mutations (V1183M, R1405K, P2266T) located respectively on NS2A, NS2B and NS4A and the "Western variant" with two nonsynonymous mutations (V1185E, V3214E) located respectively in the NS2A gene and the NS5 gene. Findings calls for in depth in vitro and functional study to elucidate the impact of observed mutations on viral fitness, spread, epidemiology and disease outcome.
\end{abstract}

Keywords: Dengue virus, complete genome, Cosmopolitan genotype, Senegal, 2018, Regional diversification

Dengue virus (DENV) a mosquito born virus is the etiological agent of dengue fever on of the most important vector-borne viral disease worldwide in term of morbidity and mortality, according to the World Health Organization (WHO) estimates (WHO, 2012). Around 3.6 billion people are at risk to dengue infection in tropical and subtropical area; each year 50200 million people are infected worldwide of which 500000 progresses to severe disease with a fatality rate of 5\% (Bhatt et al., 2013; Gubler, 1998). Infection with DENV cause clinical manifestations ranging from dengue fever with or without warning sign to life threatening disease namely severe dengue (Guzman et al., 2016).

In Senegal DENV was first reported in 1970 in Bandia area located in the region of Thies (Robin et al., 1980). The following years despite the notification of DENV-1, DENV-2 and DENV-4 the landscape of DENV circulation in Senegal was mainly dominated by the circulation of sylvatic DENV-2 in the southern part of the country (Diallo et al., 2003 ; Zeller et al., 1992). In 2009 a major change on virus epidemiology was noticed with the first urban DENV-3 outbreak in West-Africa affecting main city as Dakar, Thies and Louga (Faye et al., 2014). 
Dengue is hyperendemic (Dieng et al., 2021) in Senegal and this is known to be associated with a cyclic behaviour of serotype replacement leading to periodic epidemics (LaitonDonato et al., 2019).

Since 2017 a growing dengue incidence is noticed in Senegal with multifoci and multiserotypes circulation (Dieng et al., 2021). Interestingly, endemic behaviour may facilitate accumulation of genetic diversity and could be linked to disease spread, severity and outcomes (Laiton-Donato et al., 2019). In 2018 four dengue virus outbreaks were noticed in Senegal for two of them one in Rosso at the border with Mauritania (Manuscript in preparation) where DENV-2 was the only circulating serotype and another in Thies where DENV-3 predominant serotype co-circulated with DENV-2 (Gaye et al., 2021); in this study, we hypothesized that viral determinants have contributed to differential spread of DENV-2 during up mentioned epidemics.

We sequenced complete genomes of DENV-2 detected from suspected cases during outbreak at Rosso $(n=7)$ (Northern Senegal), Dakar $(n=2)$ (Western Senegal) and Thies $(n=1)$ (Western Senegal) in 2018. Four additional complete genome sequences from samples collected in Thies area available in GenBank NCBI were also included. Sequences were combined to available complete genome DENV-2 from West-Africa spanning the period of 2016 -2019; obtained dataset was aligned using MAFFT (Katoh et al., 2002). A Maximum Likelihood (ML) tree was drawn using IQ-TREE (Nguyen et al., 2015). Phylogenetic tree combined with Single Nucleotide Variation (SNV) of used strains in comparison to Burkina Faso strain (KY627762) was plotted; genetic distances were inferred using Mega 6 software (Tamura et al., 2013).

Performed phylogenetic analysis showed that all Senegalese DENV-2 strains belong to the Cosmopolitan genotype and are closely related to West-Africa isolates (Fourié et al., 2021). sampled between 2016 - 2019 (Supplementary figure S1; Figure 1). Interestingly, ML tree using only cluster of sequences closely related to Senegalese strains containing only West African DENV-2 sequences reveals that Senegalese sequences cluster into two different clades with a well-supported bootstrap value (Bootstrap value $=100)$; the first clade namely Northern Clade contain sequences obtained in the north of the country sampled in Rosso and Richard Toll, the second clade called Western Clade contain sequences obtained in the Western part of the country mainly during outbreak in Thies and from sporadic cases in Dakar (Figure 2). The average estimated between group mean distance was 0.53 ( $\mathrm{sd}=0.062$ ) when comparing Northern and Western clade versus $0.26(\mathrm{sd}=0.039)$ when comparing Northern Clade and group of strain from Mauritania showing that Northern Clade is closely related to isolates from Mauritania than those from the Western Clade suggesting transboundary circulation throughout a possible importation from Mauritania to Senegal or vice-versa (Figure 1; Figure 2).

Ninety-seven SNVs were observed when comparing all available West African sequences of DENV-2 with earliest DENV-2 strain from Burkina Faso (KY627762), included in the dataset, isolated in 2016. At the amino acid level, strains of Northern Clade possess three unique nonsynonymous amino acid substitution (V1183M, R1405K, P2266T) all located on Nonstructural gene in NS2A, NS2B and NS4A respectively when strains of Western Clade harbour two nonsynonymous amino acid change (V1185E, V3214E) located on NS4A and NS5 genes. For flavivirus the non-structural proteins are known to participate on critical step of viral lifecycle as replication, virion assembly and ultimately on evasion against host immune response (Zhang et al., 2019). Briefly, mutagenesis analysis study on DENV, YFV 
and WNV(Kunjin) shows that some amino acids on NS2A are essential on RNA replication (Wu et al., 2017), on virion assembly and secretion (Xie et al., 2015); for DENV NS2A antagonizes type-I IFN signalling highlighting his role on immune evasion (Muñoz-Jordan et al., 2003). DENV NS2A, 2B, 4B and NS5 inhibit IFN-mediated JAK-STAT activation and impair interferon-stimulated gene (ISG) production (Zou et al., 2019).

Functional studies on the impact of observed mutations on Senegalese strains will be crucial to decipher their role on viral spread, differential phenotypic traits (as virulence) and on virus epidemiology. 


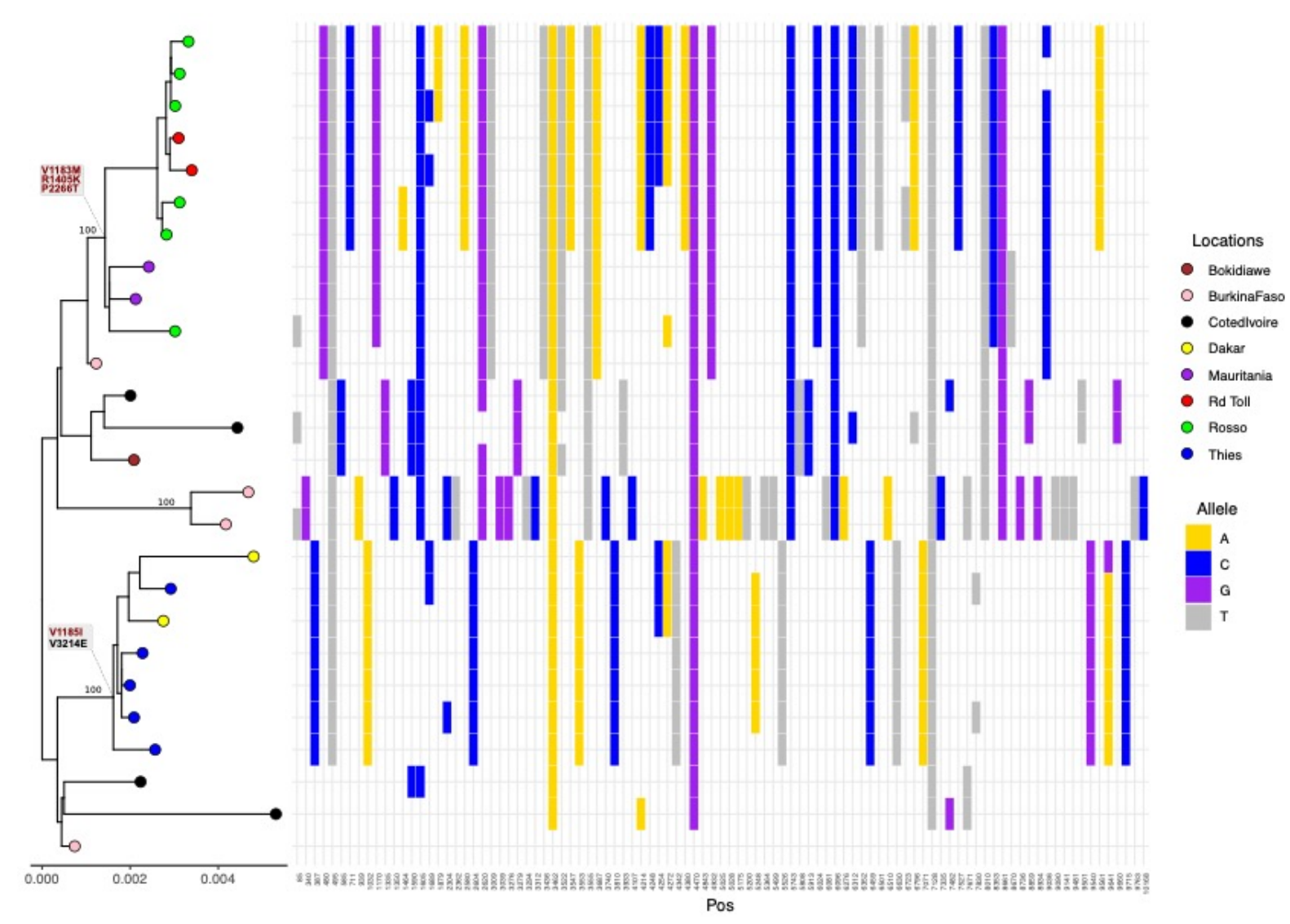

Figure 1: Phylogenetic tree based on full genome sequences of dengue virus two detected in Senegal in 2018 in combination with available sequences from Africa sampled between 20162019. Single nucleotide variation (SNV) of used sequences when compared to earliest DENV2 circulating during outbreak in Burkina Faso in 2016 (KY627762) are also represented and coloured according observed base at the given position. Site identical to the reference are not shown, changes from the Burkina Faso strain (KY627762) are indicated and coloured by nucleotide (yellow for adenosine, blue for cytosine, purple for guanine, grey for thymine). 


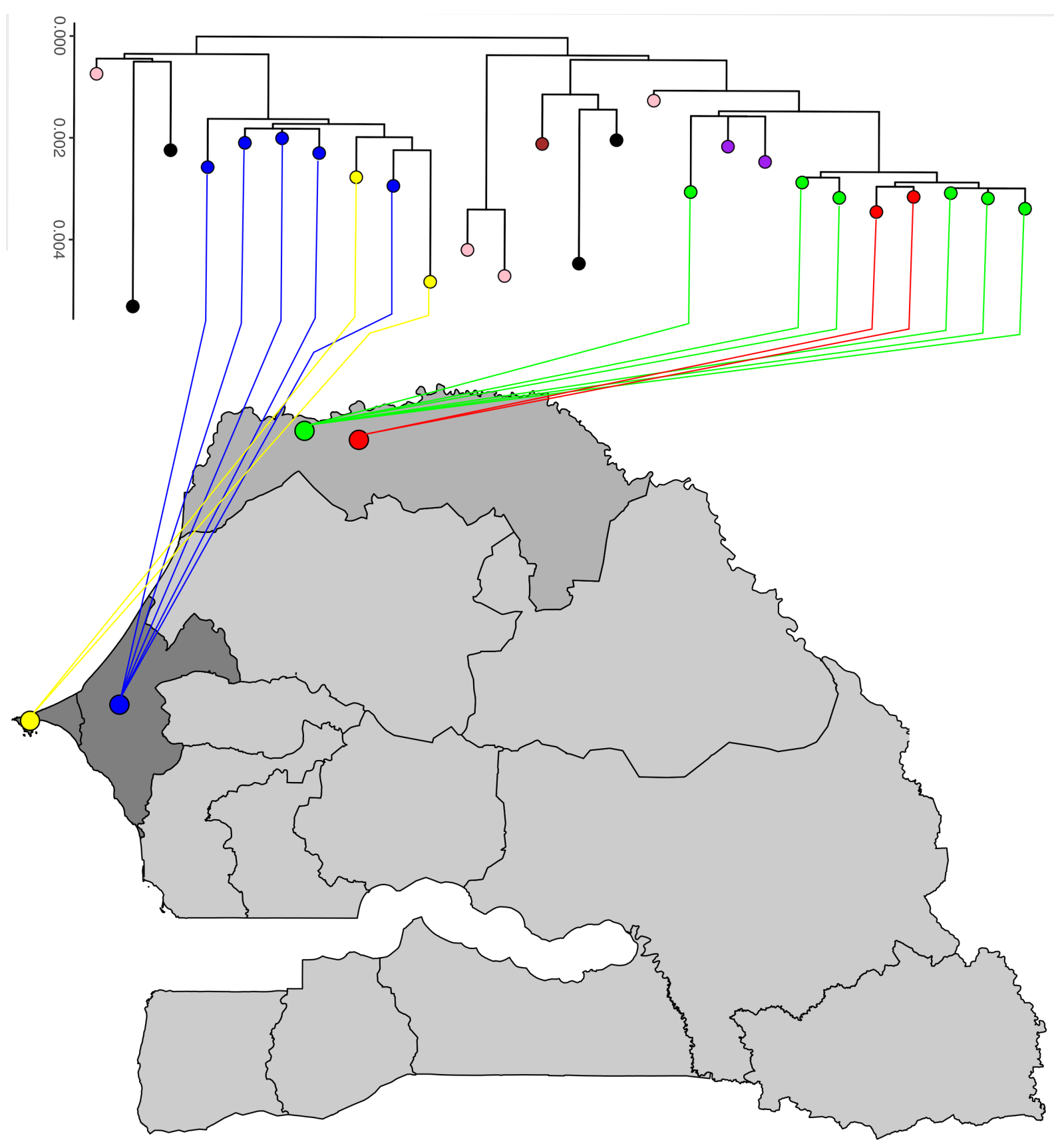

Figure 2: Map showing relationships between full genome DENV-2 from Senegal sampled in 2018 coloured by locations. Each genome is connected to the geographic location where it was collected in Senegal. Location are assigned using the same colours as on the figure 1. 


\section{Finding source}

This following study was performed using Institut Pasteur de Dakar (IPD) fund. Any special funding was received.

\section{Conflicts of interest}

The authors report no conflicts of interest

\section{Ethical Approval}

Strains used in this study are part of the Institute Pasteur in Dakar collection (WHO Collaborating Centre for Arboviruses and/or Hemorrhagic Fever Reference and Research). Samples were collected during routine surveillance. None of the data was directly derived from human or animal samples but rather from cell culture supernatant. Therefore, all the samples were anonymous and only reference numbers were used during the analysis that originated this study.

\section{Acknowledgments}

We would like to convey special thanks to the virology lab workers at the Institut Pasteur de Dakar. Special thanks to Cherif Sylla and Ndeye Amy Dieye for his awesome technical assistance. 
Bhatt, S., Gething, P. W., Brady, O. J., Messina, J. P., Farlow, A. W., Moyes, C. L., Drake, J. M., Brownstein, J. S., Hoen, A. G., Sankoh, O., Myers, M. F., George, D. B., Jaenisch, T., Wint, G. R. W., Simmons, C. P., Scott, T. W., Farrar, J. J., \& Hay, S. I. (2013). The global distribution and burden of dengue. Nature, 496(7446), 504-507. https://doi.org/10.1038/nature12060

Diallo, M., Ba, Y., Sall, A. A., Diop, O. M., Ndione, J. A., Mondo, M., Girault, L., \& Mathiot, C. (2003). Amplification of the Sylvatic Cycle of Dengue Virus Type 2, Senegal, 19992000 : Entomologic Findings and Epidemiologic Considerations. Emerging Infectious Diseases, 9(3), 362-367. https://doi.org/10.3201/eid0903.020219

Dieng, I., Ndione, M. H. D., Fall, C., Diagne, M. M., Diop, M., Gaye, A., Barry, M. A., Diop, B., Ndiaye, M., Bousso, A., Fall, G., Loucoubar, C., Faye, O., Sall, A. A., \& Faye, O. (2021). Multifoci and multiserotypes circulation of dengue virus in Senegal between 2017 and 2018. BMC Infectious Diseases, 21(1), 867. https://doi.org/10.1186/s12879-02106580-z

Faye, O., Ba, Y., Faye, O., Talla, C., Diallo, D., Chen, R., Mondo, M., Ba, R., Macondo, E., Siby, T., Weaver, S. C., Diallo, M., \& Sall, A. A. (2014). Urban Epidemic of Dengue Virus Serotype 3 Infection, Senegal, 2009. Emerging Infectious Diseases, 20(3), 456-459. https://doi.org/10.3201/eid2003.121885

Fourié, T., El Bara, A., Dubot-Pérès, A., Grard, G., Briolant, S., Basco, L. K., Ouldabdallahi Moukah, M., \& Leparc-Goffart, I. (2021). Emergence of dengue virus serotype 2 in Mauritania and molecular characterization of its circulation in West Africa. PLoS $\begin{array}{lll}\text { Neglected } \quad \text { Tropical } & \text { Diseases, } & \text { 15(10), }\end{array}$ https://doi.org/10.1371/journal.pntd.0009829 
Gaye, A., Ndiaye, T., Sy, M., Deme, A. B., Thiaw, A. B., Sene, A., Ndiaye, C., Diedhiou, Y., Mbaye, A. M., Ndiaye, I., Tomkins-Tinch, C., Gomis, J. F., Badiane, A. S., MacInnis, B., Park, D. J., Ndiaye, M., Sy, N., Sabeti, P. C., Siddle, K. J., \& Ndiaye, D. (2021). Genomic investigation of a dengue virus outbreak in Thiès, Senegal, in 2018. Scientific Reports, 11(1), 10321. https://doi.org/10.1038/s41598-021-89070-1

Gubler, D. J. (1998). Dengue and Dengue Hemorrhagic Fever. CLIN. MICROBIOL. REV., 11, 17.

Guzman, M. G., Gubler, D. J., Izquierdo, A., Martinez, E., \& Halstead, S. B. (2016). Dengue infection. Nature Reviews Disease Primers, 2(1), 16055. https://doi.org/10.1038/nrdp.2016.55

Katoh, K., Misawa, K., Kuma, K., \& Miyata, T. (2002). MAFFT: A novel method for rapid multiple sequence alignment based on fast Fourier transform. Nucleic Acids Research, 30(14), 3059-3066.

Laiton-Donato, K., Alvarez, D. A., Peláez-Carvajal, D., Mercado, M., Ajami, N. J., Bosch, I., \& Usme-Ciro, J. A. (2019). Molecular characterization of dengue virus reveals regional diversification of serotype 2 in Colombia. Virology Journal, 16(1), 62. https://doi.org/10.1186/s12985-019-1170-4

Muñoz-Jordan, J. L., Sánchez-Burgos, G. G., Laurent-Rolle, M., \& García-Sastre, A. (2003). Inhibition of interferon signaling by dengue virus. Proceedings of the National Academy of Sciences of the United States of America, 100(24), 14333-14338. https://doi.org/10.1073/pnas.2335168100

Nguyen, L.-T., Schmidt, H. A., von Haeseler, A., \& Minh, B. Q. (2015). IQ-TREE: A Fast and Effective Stochastic Algorithm for Estimating Maximum-Likelihood Phylogenies. Molecular Biology and Evolution, 32(1), 268-274. https://doi.org/10.1093/molbev/msu300 
Robin, Y., Cornet, M., Heme, G., \& Le Gonidec, G. (1980). Isolement du virus de la dengue au Sénégal. Annales de l'Institut Pasteur / Virologie, 131(2), 149-154. https://doi.org/10.1016/0769-2617(80)90029-5

Tamura, K., Stecher, G., Peterson, D., Filipski, A., \& Kumar, S. (2013). MEGA6: Molecular Evolutionary Genetics Analysis Version 6.0. Molecular Biology and Evolution, 30(12), 2725-2729. https://doi.org/10.1093/molbev/mst197

WHO. (2012). Global strategy for dengue prevention and control, 2012-2020. World Health Organization. http://apps.who.int/iris/bitstream/10665/75303/1/9789241504034_eng.pdf

Wu, R.-H., Tsai, M.-H., Tsai, K.-N., Tian, J. N., Wu, J.-S., Wu, S.-Y., Chern, J.-H., Chen, C.-H., \& Yueh, A. (2017). Mutagenesis of Dengue Virus Protein NS2A Revealed a Novel Domain Responsible for Virus-Induced Cytopathic Effect and Interactions between NS2A and NS2B Transmembrane Segments. Journal of Virology, 91(12), e01836-16. https://doi.org/10.1128/JVI.01836-16

Xie, X., Zou, J., Puttikhunt, C., Yuan, Z., \& Shi, P.-Y. (2015). Two distinct sets of NS2A molecules are responsible for dengue virus RNA synthesis and virion assembly. Journal of Virology, 89(2), 1298-1313. https://doi.org/10.1128/JVI.02882-14

Zeller, H. G., Traoré-Lamizana, M., Monlun, E., Hervy, J. P., Mondo, M., \& Digoutte, J. P. (1992). Dengue-2 virus isolation from humans during an epizootic in southeastern Senegal in November, 1990. Research in Virology, 143(2), 101-102. https://doi.org/10.1016/s0923-2516(06)80088-9

Zhang, X., Xie, X., Zou, J., Xia, H., Shan, C., Chen, X., \& Shi, P.-Y. (2019). Genetic and biochemical characterizations of Zika virus NS2A protein. Emerging Microbes $\mathcal{E}$ Infections, 8(1), 585-602. https://doi.org/10.1080/22221751.2019.1598291 
Zou, C., Huang, C., Zhang, J., Wu, Q., Ni, X., Sun, J., \& Dai, J. (2019). Virulence difference of five type I dengue viruses and the intrinsic molecular mechanism. PLoS Neglected Tropical Diseases, 13(3), e0007202. https://doi.org/10.1371/journal.pntd.0007202 